\title{
A Rare Case of a CSF Pseudocyst Mimicking a Complex Hepatic Cyst
}

\author{
Jatin Sharma Sayf Al-Katib² \\ 1Department of Diagnostic Radiology, Oakland University William \\ Beaumont School of Medicine, Royal Oak, Michigan, United States \\ ${ }^{2}$ Department of Diagnostic Radiology, William Beaumont Hospital \\ Royal Oak, Royal Oak, Michigan, United States
}

\begin{abstract}
Address for correspondence Jatin Sharma, MD, Department of Diagnostic Radiology, Oakland University William Beaumont School of Medicine, 3601 West 13 Mile Road, Royal Oak, MI 48073, United States (e-mail: Jatin.Sharma@beaumont.org, sayf.al-katib@beaumont.org).
\end{abstract}

J Gastrointestinal Abdominal Radiol ISGAR 2021;4:62-65.
Abstract
Keywords
- CSF pseudocyst
- ventriculoperitoneal shunt
- complex hepatic cyst
- shuntogram

Cerebrospinal fluid (CSF) peritoneal pseudocysts are a rare complication of ventriculoperitoneal shunts. They can manifest with symptoms of shunt malfunction or, more commonly, vague symptoms of abdominal discomfort such as pain or distention. The imaging features of peritoneal CSF pseudocysts may be nonspecific. In this case, we report a peritoneal CSF pseudocyst, which was initially mistaken for a complex hepatic cyst on both ultrasonography and MRI. The correct diagnosis was confirmed with a shuntogram and $\mathrm{CT}$ of the abdomen.

\section{Introduction}

Ventriculoperitoneal shunts drain into the peritoneal cavity and are commonly used in the management of hydrocephalus. Peritoneal cerebrospinal fluid (CSF) pseudocysts are a rare complication of ventriculoperitoneal shunts that occur with a frequency of 1 to $4.5 \%$ and can occur from 3 weeks to 5 years or more after shunt placement. ${ }^{1}$ Risk factors for CSF pseudocyst formation include infection, peritoneal adhesions, multiple shunt revisions, and peritonitis. Neurologic symptoms may present secondary to shunt malfunction and abdominal symptoms can occur secondary to mass effect.

\section{Case Presentation}

A 22-year-old male, with a past medical history of grand mal seizures and hydrocephalus status postventriculoperitoneal shunt placement with last revision 12 years prior, presented for gastroscopy for evaluation of gastroesophageal reflux disease. During gastroscopy, small columns of esophageal varices were identified and a follow-up abdominal US was ordered to evaluate for cirrhosis. Abdominal US did not reveal cirrhosis but noted a complex cystic lesion with internal septations believed to be arising from the left hepatic lobe ( $\sim$ Fig. 1 A, B). Subsequent abdominal contrast-enhanced abdominal MRI confirmed the presence of a complex cystic lesion believed to be arising from the posterior left hepatic lobe, with thin nonenhancing septations and areas of internal proteinaceous

published online October 12, 2020
DOI https://doi.org/ $10.1055 / \mathrm{s}-0040-1716788$ ISSN 2581-9933. material. The differential diagnosis included biliary cystadenoma, biliary cystadenocarcinoma, and an epithelial cyst complicated by prior infection/hemorrhage. The patient was referred to a hepatic surgeon who recommended a follow-up MRI in 3 months.

The patient presented to the emergency department 2 months later for headaches and nausea. Head CT and radiographic ventriculoperitoneal shunt surveys at this time were unremarkable for acute abnormalities. The follow-up MRI abdomen was performed, and this time it was suggested that the cyst was intraperitoneal in location and separate from the liver. Also, the internal septations showed signal void on all sequences, suggesting that they represent tubing from the ventriculoperitoneal shunt catheter ( $\mathbf{- F i g .} 2 \mathrm{~A}-\mathbf{F}$ ). The diagnosis of CSF pseudocyst was suggested. The referring consultant was of the opinion that the patient's neurologic symptoms were likely the result of shunt malfunction and that laparoscopic lysis of adhesions would be considered if symptoms persisted.

The patient subsequently presented to the emergency department 2 months later for acute onset of dizziness and syncope. Initial physical examination, CT head, and ventriculoperitoneal shunt surveys ( - Fig. 3 A, B) revealed no acute abnormalities. Radiographic shuntogram with injection of $3 \mathrm{~mL}$ contrast through the shunt tubing ( - Fig. 4) and subsequent CT abdomen ( - Fig. 5 A, B) revealed contrast confined within the peritoneal pseudocyst. Intracranial pressures were with within normal limits and fluid analysis revealed

(C)2020. Indian Society of Gastrointestinal and Abdominal Radiology. This is an open access article published by Thieme under the terms of the Creative Commons Attribution-NonDerivative-NonCommercial-License, permitting copying and reproduction so long as the original work is given appropriate credit. Contents may not be used for commercial purposes, or adapted, remixed, transformed or built upon. (https://creativecommons.org/licenses/by-nc-nd/4.0/).

Thieme Medical and Scientific Publishers Pvt. Ltd. A-12, 2nd Floor, Sector 2, Noida-201301 UP, India 


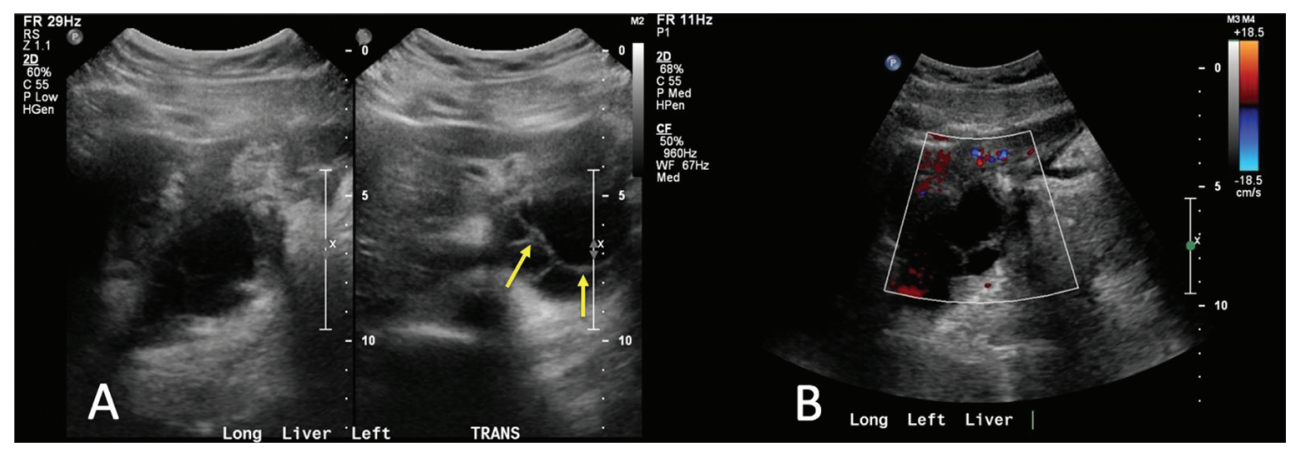

Fig. 1 Greyscale (A) and color Doppler (B) ultrasound shows a circumscribed cystic collection with thin septations (arrows). The collection contacts the posterior wall of the left hepatic lobe. No internal flow is seen on Doppler (B).

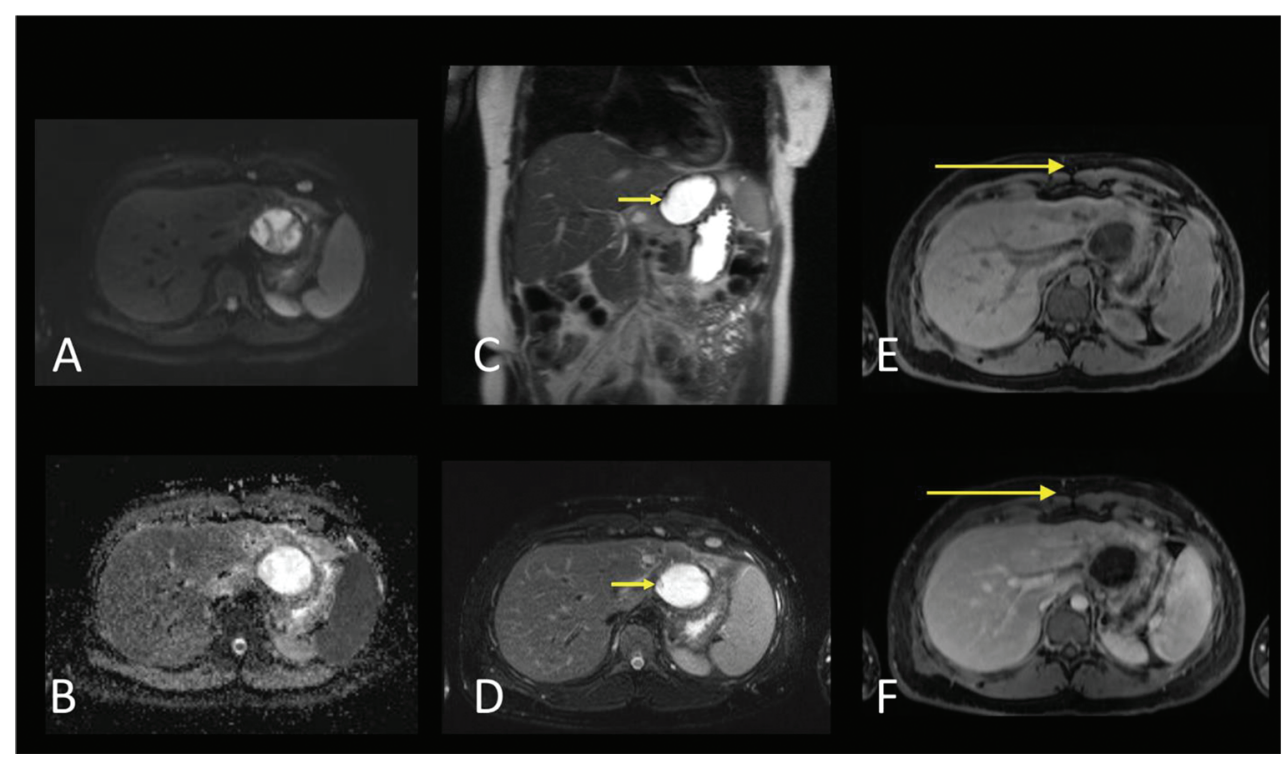

Fig. 2 MRI demonstrates the T2 hyperintense gastrohepatic collection. There is no diffusion restriction on diffusion (A) and apparent diffusion coefficient (ADC) (B) sequences. There are nonenhancing "septations" along the medial wall (C, D) which represented catheter tubing material. Very faintly, visible shunt catheter tubing can be seen in the subcutaneous tissues of the anterior abdominal wall (E, F).

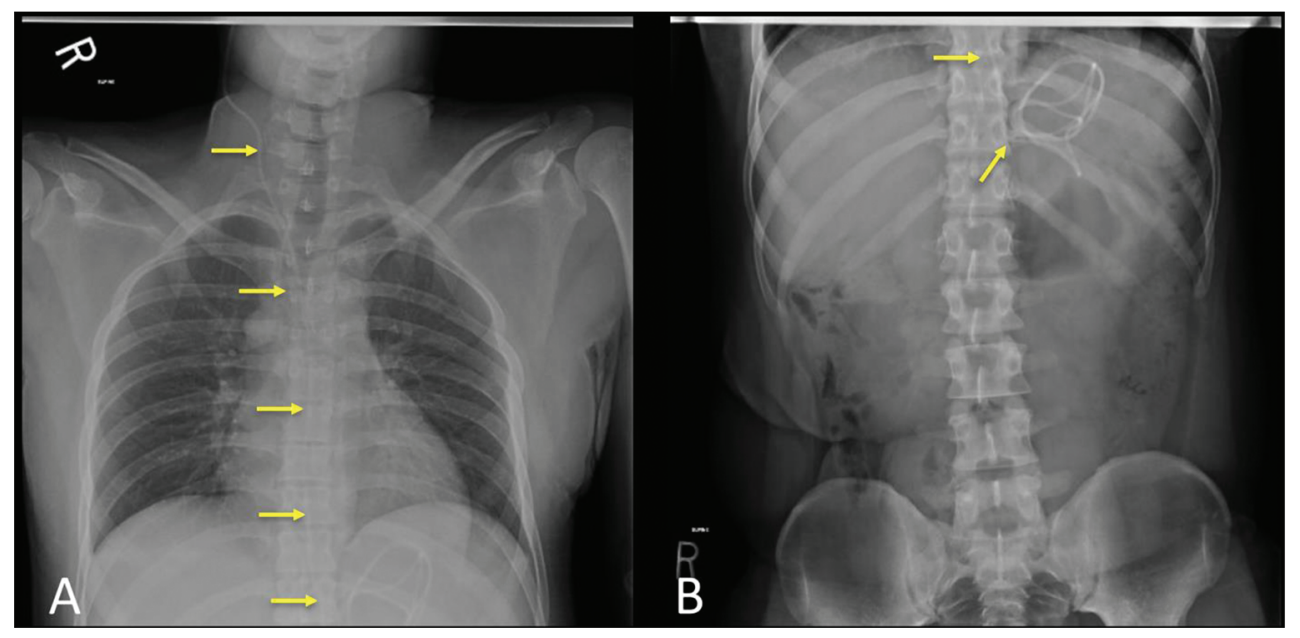

Fig. 3 Frontal chest (A) and abdomen (B) radiographs demonstrate ventriculoperitoneal shunt tubing (arrows) coursing along the right neck and chest wall and curled within the left upper quadrant of the abdomen in the expected location of the cystic collection. 
no signs of infection. The neurosurgery team concluded that the shunt did not need revision. The patient had no further episodes of syncope and was discharged with antiepileptic medication.

\section{Discussion}

Peritoneal CSF pseudocyst formation is a rare complication of ventriculoperitoneal shunts. These pseudocysts are surrounded by a wall of nonepithelial tissue and filled with CSF. On histology, they show fibrous tissue lined with collagen, fibroblasts, inflammatory cells, and granulomatous tissue within the wall. While the exact pathophysiology is unknown, it is theorized that the nonepithelial lining and

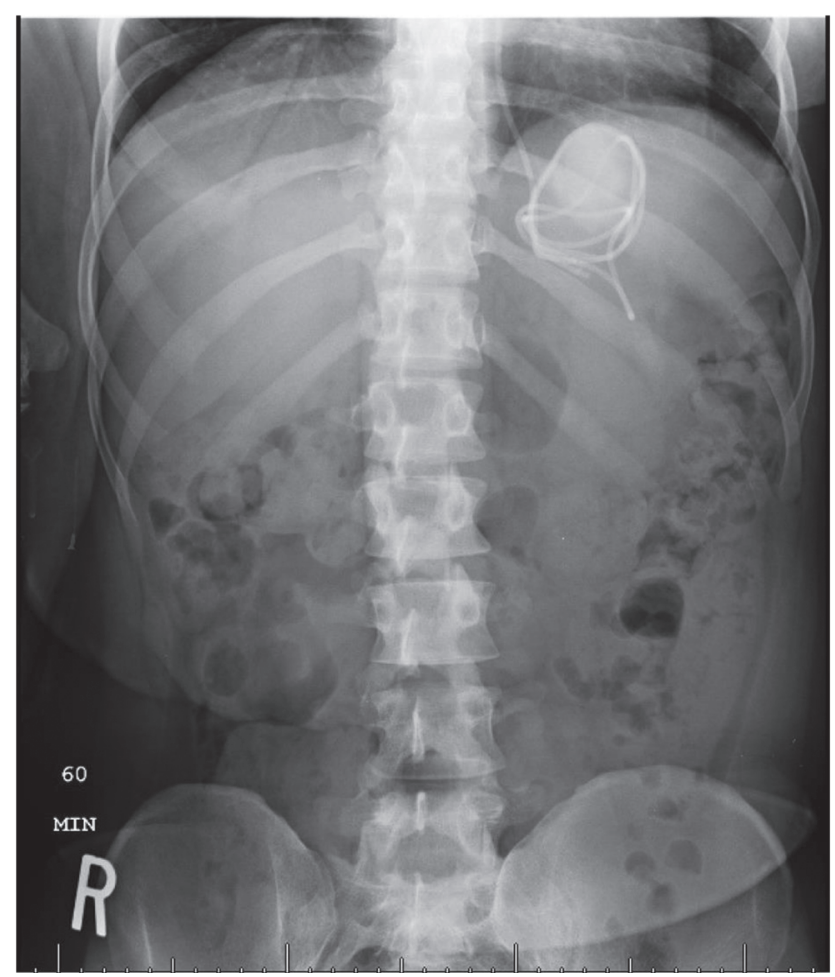

Fig. 4 Postshuntogram frontal radiograph of the abdomen shows collection of contrast at the region of the distal ventriculoperitoneal shunt without spill into the remainder of the peritoneum. inflammatory cells inhibit CSF absorption and can impair drainage. ${ }^{2}$ Predisposing factors include proinflammatory processes such as infection, multiple shunt revisions, peritonitis, and adhesions. ${ }^{2}$ In our case, the patient had a history of 23 prior shunt revisions.

Peritoneal pseudocysts can increase in size, resulting in abdominal pain, distention, bowel obstruction, and acute abdomen secondary to mass effect. ${ }^{3}$ CSF pseudocysts can also disrupt ventriculoperitoneal shunt function, leading to headaches, nausea, and symptoms of increased intracranial pressure. In our case, the CSF pseudocyst was the presumed source of the patient's dizziness, syncope, and headaches; however, neuroimaging failed to reveal any complications or new findings when compared with previous imaging.

The case highlights the shortcomings of US and MRI in the assessment of CSF pseudocysts, as shunt catheter tubing is not readily identifiable on these imaging modalities. Conventional radiography may suggest the diagnosis by showing the distal catheter coiling within the abdomen. ${ }^{4} \mathrm{CT}$ most optimally images the distal catheter, and in cases of CSF pseudocyst formation, can readily identify any surrounding fluid collection. ${ }^{4}$

In our case, the "septations" identified on the original abdominal US were, in fact, looped portions of the distal shunt catheter. A subsequent MRI also misinterpreted the internal signal voids to represent nonenhancing septations. While the cyst contacted the posterior left hepatic lobe, it was, in fact, arising from the peritoneum rather than from the liver. On a follow-up MRI, the shunt catheter tubing could be vaguely seen in the anterior abdominal wall, extending toward the fluid collection which helped point to the correct diagnosis.

Subsequent shuntogram showed the distal ventriculoperitoneal catheter tubing coiling in the left upper abdomen. Upon injection of contrast, there was pooling of contrast surrounding the distal shunt tubing without spill into the remainder of the peritoneal cavity. Non-IV contrast CT of the abdomen confirmed contrast material confined to the fluid collection in the gastrohepatic space surrounding the distal shunt tubing. As this case demonstrates, it is important to keep peritoneal pseudocysts in the differential diagnosis when evaluating patients with ventriculoperitoneal catheters, as their imaging findings can be overlooked.

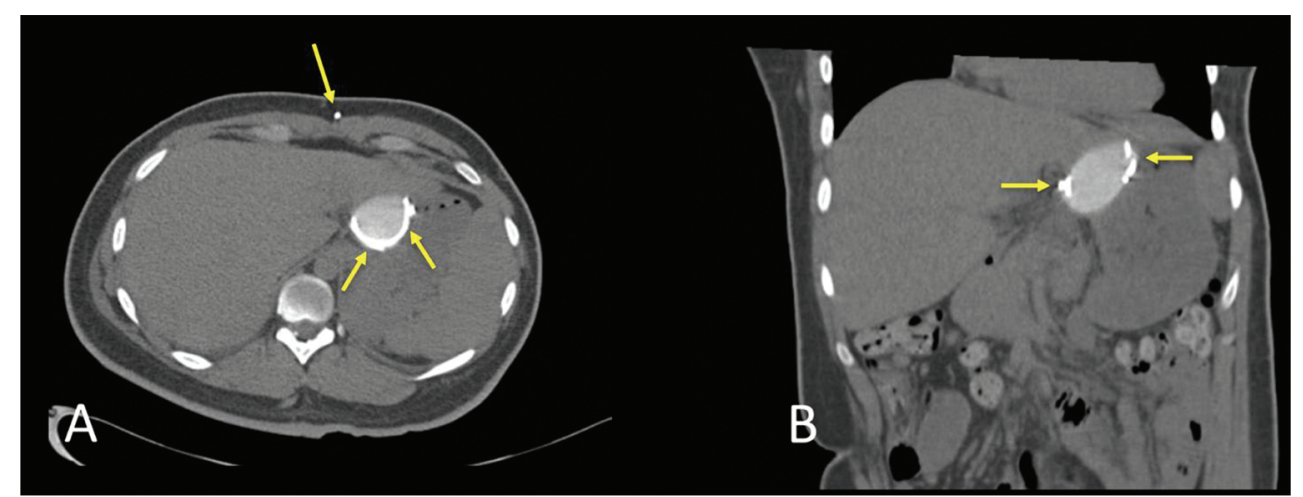

Fig. 5 Axial (A) and coronal (B) unenhanced CT of the abdomen demonstrates radiopaque shunt catheter tubing (arrows) curled within the contrast opacified collection in the gastrohepatic region. 
Management of CSF pseudocysts is highly variable and dependent on whether shunt malfunction or symptoms are present. Pseudocysts can be conservatively monitored if they are not interfering with shunt function or causing abdominal symptoms. Symptomatic pseudocysts are amenable to percutaneous drainage, repositioning of the distal peritoneal catheter, laparoscopic lysis of adhesions, or may require complete excision of pseudocyst and removal of ventriculoperitoneal shunt hardware. ${ }^{5}$ In our case, the patient did have a shunt revision 2 years following this admission with subsequent resolution of the pseudocyst.

\section{Conclusion}

CSF peritoneal pseudocysts are rare complications of ventriculoperitoneal shunt catheter placement and are important to keep in the differential diagnosis of intraperitoneal cystic lesions in these patients. CSF pseudocysts should be considered in patients with ventriculoperitoneal shunt, who present with nonspecific abdominal symptoms or neurologic symptoms suggestive of shunt malfunction. This case demonstrates how US and MRI do not optimally image shunt catheter tubing and therefore suggests alternative diagnoses. CT more optimally images distal shunt catheter tubing as well as surrounding fluid collections and is better able to suggest the diagnosis of CSF pseudocyst. Radiographic shuntogram may also more clearly demonstrate pooling of contrast surrounding distal catheter tubing and aid in confirming the diagnosis.

\section{Funding}

None.

\section{Conflict of Interest}

None declared.

\section{References}

1 Erşahin Y, Mutluer S, Tekeli G. Abdominal cerebrospinal fluid pseudocysts. Childs Nerv Syst 1996;12(12):755-758

2 Hamid R, Baba AA, Bhat NA, Mufti G, Mir YA, Sajad W. Post ventriculoperitoneal shunt abdominal pseudocyst: challenges posed in management. Asian J Neurosurg 2017;12(1):13-16

3 Oxenberg J. Giant intraperitoneal multiloculated pseudocyst in a male. Case Rep Surg 2016 (e-pub ahead of print). doi:10.1155/2016/4974509

4 Wallace AN, McConathy J, Menias CO. Bhalla S, Wippold FJ II. Imaging evaluation of CSF shunts. AJR Am J Roentgenol 2014;202(1):38-53

5 Kashyap S, Ghanchi H, Minasian T, Dong F, Miulli D. Abdominal pseudocyst as a complication of ventriculoperitoneal shunt placement: Review of the literature and a proposed algorithm for treatment using 4 illustrative cases. Surg Neurol Int 2017;8(1):78 